\title{
Zur N-reduzierten Fütterung von Mastschweinen bei Einsatz freier Aminosäuren
}

\begin{abstract}
Summary
Title of the Paper: Investigation on N-reduced feeding and use of free amino acids in fattening pigs The aim of the present study was to investigate the $\mathrm{N}$-reduced feeding by fattening pigs feed by cereals rich feedmixture supplemented with the free amino-acids L-lysine, DL-methionine, L-threonine, L-tryptophane, Lleucine, L-isoleucine, L-histidine and L-valine. It was to test extreme low crude protein content by requirement sufficient supply on amino-acid. The reaction of the animals in growth, carcass quality and the reduction of $\mathrm{N}$ excretion was to investigate.

The pigs were divided in two groups (control and experimental group) with 24 animals per group (12 females and 12 castrates). The experiment was divided into three periods ( $25-60,60-85$ and $85-110 \mathrm{~kg}$ body weight). The protein content of the feedmixture was by the control group 18.3, 15.6 and $13.6 \%$ and by the experimental group 14.0, 11.8 and $10.7 \%$, respectively. The lysine-energy ratio of the feedmixture was by all three feeding periods by 0.72 or $0.74,0.61$ or 0.62 and 0.55 or $0.57 \mathrm{~g}$ lysine per MJ ME in control feedmixture or experimental feedmixture, respectively.

By a high performance are the average daily life weight gain (control group 869 and experimental group $863 \mathrm{~g}$ ) and the lean meat rate (control group 55.2 and experimental group 55:0\%) not influenced by the high of crude protein supply. The calculated $\mathrm{N}$-excretion were by the control group $4.16 \mathrm{~kg}$ (as $100 \%$ ) and by experimental group $2.60 \mathrm{~kg}(62.5 \%)$ per $100 \mathrm{~kg}$ body weight gain.
\end{abstract}

Key Words: pig fattening, protein reduced diets, amino acid supplementation, growth performance, carcass quality, $\mathrm{N}$ excretion

\section{Zusammenfassung}

Es wird uber Untersuchungen zur N-reduzierten Fütterung von Mastschweinen bei Einsatz getreidereicher Futtermischungen und Supplementierung mit den freien Aminosäuren L-Lysin, DL-Methionin, L-Threonin, LTryptophan, L-Leucin, L-Isoleucin, L-Histidin und L-Valin berichtet. Es sollte gepruft werden, ob eine extreme Absenkung des Rohproteingehaltes bei Gewăhrleistung einer bedarfsgerechten Aminosăurenversorgung der Tiere Auswirkungen auf Wachstum und Schlachtkőrperqualităt hat und in welchem Umfang die NAusscheidungen reduziert werden können. Die Untersuchungen umfaßten zwei Fütterungsgruppen (Versuchsund Kontrollgruppe) mit jeweils 24 Tieren (12 Börge und 12 weibliche Tiere) und drei Mastabschnitte (25-60, 60-85, 85-110 kg LM). Die Rohproteingehalte der Futtermischungen betrugen bei der Kontrollgruppe 18,3; 15,6 sowie $13,6 \%$ und bei der Versuchsgruppe 14,$0 ; 11,8$ sowie $10,7 \%$. Die Lysin/Energie-Verhăltnisse der Futtermischungen waren für die drei Mastabschnitte bei 0,72 bzw. 0,$74 ; 0,61$ bzw. 0,62 sowie 0,55 bzw.57 g Lysin je MJ ME eingestellt.

Bei hohem Leistungsniveau sind die mittleren tăglichen Lebendmassezunahmen je Tier (Kontrollgruppe: 869 g; Versuchsgruppe: $863 \mathrm{~g}$ ) sowie die Magerfleischanteile (Kontrollgruppe: 55,2\%; Versuchsgruppe: 55,0\%) durch die Høhe der Rohproteinversorgung nicht beeinflußt worden. Die kalkulierten N-Ausscheidungen lagen bei der Kontrollgruppe bei $4,16 \mathrm{~kg}(100 \%)$ und bei der Versuchsgruppe bei $2,60 \mathrm{~kg}(62,5 \%)$ je100 $\mathrm{kg}$ Lebendmassezunahme.

Schlusselwðrter: Schweinemast, Rohproteinreduzierung, Aminosăurensupplementierung, Wachstumsleistung, Schlachtkörperqualităt, N-Ausscheidungen 
1. Einleitung

Mit vorliegendem Beitrag sollen bestehende Möglichkeiten einer proteinreduzierten Fütterung von Mastschweinen bei Einsatz getreidereicher Futtermischungen weiter ausgelotet werden. Die dafür durchgeführten Untersuchungen stellen eine Fortsetzung der Arbeit von NONN und FRANKE (1998) dar. Bei dieser war, bezugnehmend auf Bilanzstudien von KIRCHGESSNER et al. (MARKERT, 1992; MARKERT et al., 1993; ROTH et al., 1994; KIRCHGESSNER et al., 1994) sowie auf die ideale Aminosäurenzusammensetzung des Futterproteins nach WANG und FULLER (1990), der Rohproteingehalt von getreidereichen Futtermischungen in vier Abstufungen von $18 \%$ auf $13 \%$ im Lebendmasseabschnitt von $28-55 \mathrm{~kg}$ und von $16 \%$ auf $11,5 \%$ im Lebendmasseabschnitt von 55-110 kg abgesenkt und die Versorgung mit den Aminosäuren Lysin, Methionin, Threonin und Tryptophan durch deren Supplementierung als freie Aminosäuren bedarfsdeckend gestaltet worden. Dabei zeigte sich bis zu einer Absenkung der Rohproteingehalte auf $15 \%$ (LM 28-55 kg) bzw. $13 \%$ (LM 55-110 kg) keine spürbare Beeinträchtigung der Wachstumsleistungen und Schlachtkörperqualität bei einer Verringerung der $\mathrm{N}$-Ausscheidungen je $\mathrm{kg}$ Lebendmassezunahme um ca. $30 \%$. Die weitere Absenkung des Rohproteingehaltes in den Futtermischungen auf 13,5 \% (LM 28-55 kg) und 11,5\% (LM 55-110 kg) führte allerdings zu negativen Folgen für das Wachstum und die Schlachtkörperqualität. Als Grund wird dafür eine partielle Unterversorgung der Versuchstiere mit den Aminosäuren Isoleucin, Leucin und Valin angesehen.

Bei der Versuchsanstellung zu vorliegendem Beitrag wurde unter Beachtung neuerer Untersuchungsbefunde zum Aminosäurenbedarf sowie zur idealen Amiosäurenzusammensetzung des Futterproteins (COLE und VAN LUNEN, 1994 ; SUSENBETH et al., 1994; KERR et al., 1995; HAHN und BAKER, 1995; BAKER, 1997) und durch Supplementierung mit weiteren essentiellen Aminosäuren (gegebenenfalls aller essentieller Aminosäuren) der Proteingehalt in den Futtermischungen der Versuchsgruppe weiter abgesenkt. Außerdem wurde von einer zweiphasigen auf eine dreiphasige Fütterung umgestellt, wodurch noch eine genauere Anpassung der Aminosäurenversorgung an den Bedarf erreicht werden sollte. Andererseits wurde bei vorliegenden Untersuchungen die Verdaulichkeit der Rohnährstoffe nicht bestimmt, so daß die Energiedichte der Futtermischungen nur auf der Basis der Rohnährstoffgehalte geschätzt werden konnte.

2. Material und Methoden

Der Versuch umfaßte zwei Fütterungsgruppen (Kontrollgruppe KG und Versuchsgruppe VG). Die Versuchstiere waren Hybriden aus der Anpaarung von Deutschem Edelschwein [Mutter] x Pietrain [Vater] bzw. Landrasse x Dt. Edelschwein [Mutter] x Pietrain [Vater], die zu Versuchsbeginn eine Lebendmasse von $26,5 \pm 3,2 \mathrm{~kg}$ aufwiesen. Der Mastversuch war in drei Abschnitte (26-60 kg LM, 60-85 kg LM und 85-110 kg LM - 3-Phasen-Fütterung) untergliedert. Bei der Kontrollgruppe wurden Rohproteingehalte von $18,3 \% ; 15,5 \%$ und $13,6 \%$ in den Futtermischungen vorgesehen, die den Empfehlungen des DLG-Arbeitskreises Futter und Fütterung (1995) für Alleinfutter für Mastschweine (Dreiphasenfütterung, Energiekonzentration 13,0 MJ ME/kg) 
Entsprechen, und bei der Versuchsgruppe die Rohproteingehalte auf 14,0 \%, 11,5 und $10,5 \%$ (für die drei Lebendmasseabschnitte) in den Futtermischungen abgesenkt. Die Futtermischungen basierten vorrangig auf Getreide und sollten einen einheitlichen Energiegehalt von 13,0 MJ ME/kg aufweisen. Es wurde von den Lysinversorgungsempfehlungen der Gesellschaft für Ernährungsphysiologie (GfE, 1987) ausgegangen. Danach ergibt sich der Lysinbedarf aus der Gleichung:

Lysinbedarf g/Tier/d $=2,970 \mathrm{~W}+0,0176 \Delta \mathrm{W}-0,0059 \mathrm{~W}^{2}-8,2143 \mathrm{~W}^{0,75}$

(W = Lebendmasse)

Bei Unterstellung eines mittleren Futterverzehrs und hoher Wachstumsleistung folgen daraus Lysingehalte in den Futtermischungen von 0,97 \% (28-60 kg LM), 0,82\% (60$85 \mathrm{~kg} \mathrm{LM}$ ) sowie 0,72 \% (85-110 kg LM). Darauf Bezug nehmend, ist für die Versorgung mit den übrigen essentiellen Aminosäuren die ideale Proteinzusammensetzung nach CHUNG und BAKER (1992) zugrunde gelegt worden. Die daraus abgeleiteten angestrebten Mindestgehalte an essentiellen Aminosäuren in den Futtermischungen sind in Tabelle 1 ausgewiesen.

Tabelle 1

Angestrebte Mindestgehalte essentieller Aminosäuren in den Futtermischungen (\%) (Striven base content on amino acids in feed mixtures (\%))

\begin{tabular}{lccc}
\hline & & Lebendmasse (kg/Tier) & \\
& $25-60$ & $60-85$ & $85-110$ \\
\hline Lysin & 0,97 & 0,82 & 0,72 \\
Met + Cys & 0,60 & 0,53 & 0,46 \\
Methionin & 0,35 & 0,29 & 0,25 \\
Threonin & 0,65 & 0,57 & 0,50 \\
Tryptophan & 0,18 & 0,16 & 0,14 \\
Leucin & 0,97 & 0,82 & 0,72 \\
Isoleucin & 0,58 & 0,49 & 0,43 \\
Histidin & 0,31 & 0,26 & 0,23 \\
Phenylalanin & 0,56 & 0,48 & 0,41 \\
Phe + Tyr & 0,92 & 0,78 & 0,68 \\
Valin & 0,66 & 0,56 & 0,49 \\
Arginin & 0,65 & 0,57 & 0,48 \\
\hline
\end{tabular}

Die Futtermischungen der Kontrollgruppe wurden auf Getreidebasis mit Proteinergänzung durch Soja- und Rapsextraktionsschrot erstellt. Sie erhielten bereits eine geringe Supplementierung mit Lysin, Methionin und Threonin als freie Aminosäuren. Bei den Futtermischungen der proteinreduzierten Versuchsgruppe wurden Soja- und Rapsextraktionsschrot stark reduziert und die Bedarfsdeckung aller essentiellen Aminosäuren durch deren Supplementierung gewährleistet. Als freie Aminosäuren kamen LysinHCL (L-Lysingehalt 78 \%), DL-Methionin, L-Threonin und L-Tryptophan (jeweils 98\% AS-Gehalt) als zugelassene Handelspräparate sowie L-Leucin, L-Isoleucin, LHistidin und L-Valin (jeweils $98 \%$ AS-Gehalt) als Versuchspräparate der Firma Eurolysin zum Einsatz.

In Tabelle 2 sind Komponenten und in Tabelle 3 die analysierten Nährstoffgehalte der Futtermischungen ausgewiesen.

Die Rohnährstoff-, Stärke- und Zuckergehalte der Futtermischungen wurden nach verbindlichen VDLUFA-Methoden (NAUMANN und BASSLER, 1993) und die Amino- 
säuren mittels Ionenaustausch-Chromatographie nach BASSLER und BUCHHOLZ (1993) bestimmt. In Tabelle 3 sind außerdem die Energiedichten der Futtermischungen ausgewiesen, die auf der Basis der analysierten Rohnährstoffe nach der Gleichung (GfE, 1987)

$$
\begin{aligned}
\mathrm{ME}(\mathrm{MJ} / \mathrm{kg})= & 0,0223^{*} \mathrm{~g} \text { Rohprotein }+0,0341^{*} \mathrm{~g} \text { Rohfett }+0,017 * \mathrm{~g} \text { Stärke } \\
& +0,0144^{*} \mathrm{~g} \text { Zucker }+0,0074^{*} \mathrm{~g} \text { organischer Rest }-0,0109^{*} \mathrm{~g} \text { Rohfaser }
\end{aligned}
$$

ermittelt worden sind. Der organische Rest stellt die Differenz zwischen organischer Substanz und der Summe aus Rohprotein, Rohfett, Stärke, Zucker und Rohfaser dar. Weiterhin enthält Tabelle 3 Angaben über die Rohprotein/Energie- bzw. Ly$\sin$ /Energierelationen in den Futtermischungen.

Es wird ersichtlich, daß die angestrebten Gehalte der Futtermischungen an Rohprotein und Aminosäuren, die supplementiert wurden, mit akzeptabler Genauigkeit realisiert werden konnten.

Tabelle 2

\begin{tabular}{|c|c|c|c|c|c|c|}
\hline \multirow[b]{2}{*}{ Lebendmasse (BW) kg } & \multicolumn{3}{|c|}{ Kontrollgruppe } & \multicolumn{3}{|c|}{ Versuchsgruppe } \\
\hline & $25-60$ & $60-85$. & $85-110$ & $25-60$ & $60-85$ & $85-110$ \\
\hline CP-Gehalt im Futter $\mathrm{g} / \mathrm{kg}$ & 183 & 156 & 136 & 140 & 118 & 107 \\
\hline \multicolumn{7}{|l|}{ Komponenten (Ingred.) } \\
\hline Gerste (Barley) & 40,0 & 35,0 & 42,0 & 34,0 & 10,0 & 39,0 \\
\hline Weizen (Wheat) & 32,3 & 37,22 & 33,14 & 50,57 & 48,31 & 34,3 \\
\hline Mais (Maize) & & 7,0 & 6,0 & & 30,0 & 18,0 \\
\hline $\begin{array}{l}\text { Sojaextraktionsschrot } \\
\text { (Soybean meal) }\end{array}$ & 16,0 & 9,0 & 7,0 & 2,5 & & \\
\hline $\begin{array}{l}\text { Rapsextraktionsschrot } \\
\text { (Rape seed meal) }\end{array}$ & 5,0 & 5,0 & 5,0 & 5,0 & 4,0 & 1,0 \\
\hline Melasse & 2,0 & 2,0 & 2,0 & 2,0 & 2,0 & 2,0 \\
\hline Rapsøl & 2,0 & 2,0 & 2,0 & 2,0 & 2,0 & 2,0 \\
\hline $\begin{array}{l}\text { Mineralfutter } \\
\text { (Mineral mix.) }\end{array}$ & 2,5 & 2,5 & 2,5 & 2,5 & 2,5 & 2,5 \\
\hline Lysin-HCL & 0,14 & 0,24 & 0,28 & 0,64 & 0,62 & 0,6 \\
\hline DL-Methionin & 0.06 & 0,04 & 0,04 & 0,14 & 0,10 & 0,12 \\
\hline L-Threonin & & & 0,04 & 0,23 & 0,21 & 0,21 \\
\hline L-Tryptophan & & & & 0,3 & 0,05 & 0,05 \\
\hline L-Leucin & & & & 1,0 & & \\
\hline L-Isoleucin & & & & 1,9 & 0,14 & 0,12 \\
\hline L- Histidin & & & & 0,2 & & 0,01 \\
\hline L-Valin & & & & 0,8 & 0,07 & 0,09 \\
\hline
\end{tabular}

Zusammensetzung der Futtermischungen (\%) (Composition of the diets (\%))

Je Fütterungsgruppe wurden 24 Tiere - 12 weibliche (S; Sauen) und 12 männliche Kastraten (B; Börge) - verwendet. Die Zuordnung der Tiere in die Fütterungsgruppen wurde unter Berücksichtigung von Abstammung und Lebendmasse vorgenommen. Die Lebendmassevariation lag zu Versuchsbeginn zwischen den Varianten bei $< \pm 0,6$ $\mathrm{kg}$. Die Tiere wurden in Einzelbuchten $(2 \mathrm{~m} \mathrm{x} \mathrm{0,85} \mathrm{m)} \mathrm{mit} \mathrm{Einzelfütterung} \mathrm{auf} \mathrm{Voll-}$ spaltenboden gehalten. Die Wasserversorgung erfolgte durch Nippeltränken. Die weiblichen Tiere wurden während der gesamten Mastdauer ad libitum, die Börge bis $60 \mathrm{~kg}$ Lebendmasse ad libitum und danach restriktiv mit 2,8 kg Futterzuteilung je Tag $(1,4 \mathrm{~kg} /$ Mahlzeit) gefüttert. Es gab täglich zwei Futterzuteilungen, die bei Ad-libitum- 
Fütterung am Verzehr des Vortages orientiert waren. Die Stalltemperatur lag während des Versuches im Bereich von 16-21 ${ }^{\circ} \mathrm{C}$ und die Lufteuchte zwischen 75 und $85 \%$. Bei Bedarf wurde der Stall geheizt. Die Einstallung erfolgte Mitte September 1998. Die Tiere wurden im 14tägigen Abstand gewogen und der Futterverzehr für jedes Einzeltier wöchentlich erfaßt. Alle Untersuchungsbefunde sind geschlechtsspezifisch ausgewertet worden.

Die N-Ausscheidungen wurden anhand der Kenndaten des Wachstumsversuches kalkuliert. Für die Berechnung des Proteinansatzes kam dabei die von der GfE (1987) zur Proteinbedarfsableitung benutzte Formel:

$$
\text { Proteinansatz }=5,73 \mathrm{~W}^{0,75}-0,1513 \mathrm{~W}^{1,5}+0,11 \Delta \mathrm{W}
$$

zur Anwendung.

Tabelle 3

Rohnăhrstoff-, Aminosăuren- und Energiegehalte in Futtermischungen (\%) (Contens of crude nutrients, amino acids and energie in the diets for (\%))

\begin{tabular}{|c|c|c|c|c|c|c|}
\hline \multirow[b]{2}{*}{ Lebendmasse $\mathrm{kg} / \mathrm{Tier}$} & \multicolumn{3}{|c|}{ Var. I (Kontrollgruppe) } & \multicolumn{3}{|c|}{ Var. II (Versuchsgruppe) } \\
\hline & $25-60$ & $60-85$ & $85-110$ & $25-60$ & $60-85$ & $85-110$ \\
\hline CP-Gehalt im Futter $\mathrm{g} / \mathrm{kg}$ & 183 & 156 & 136 & 140 & 118 & 107 \\
\hline \multicolumn{7}{|l|}{ Inhaltsstoffe (Analy.) } \\
\hline Rohprotein (CP) & 18,33 & 15,57 & 13,61 & 13,97 & 11,79 & 10,67 \\
\hline Rohfett (EE) & 3,4 & 3,6 & 3,5 & 3,6 & 4,1 & 4,1 \\
\hline Rohfaser (CF) & 3,5 & 3,4 & 3,6 & 3,6 & 3,2 & 3,1 \\
\hline Rohasche (TÁ) & 0,6 & 0,5 & 0,5 & 0,4 & 0,5 & 0,4 \\
\hline Stärke (Starch) & 38,4 & 43,2 & 39,1 & 40,0 & 43,8 & 44,6 \\
\hline Zucker (Sugar) & 4,5 & 4,0 & 3,0 & 3,6 & 3,3 & 3,8 \\
\hline Lysin & 0,98 & 0,86 & 0,73 & 0,97 & 0,82 & 0,73 \\
\hline Met + Cys & 0,68 & 0,59 & 0,54 & 0,62 & 0,54 & 0,47 \\
\hline Methionin & 0,35 & 0,29 & 0,25 & 0,35 & 0,29 & 0,25 \\
\hline Threonin & 0,70 & 0,58 & 0,51 & 0,65 & 0,58 & 0,51 \\
\hline Tryptophan & 0,22 & 0,18 & 0,16 & 0,18 & 0,17 & 0,14 \\
\hline Leucin & 1,35 & 1,14 & 1,01 & 0,98 & 0,91 & 0,77 \\
\hline Isoleucin & 0,64 & 0,52 & 0,49 & 0,59 & 0,50 & 0,43 \\
\hline Histidin & 0,44 & 0,36 & 0,33 & 0,31 & 0,26 & 0,23 \\
\hline Phenylalanin & 0,91 & 0,78 & 0,73 & 0,61 & 0,54 & 0,52 \\
\hline Phe + Tyr & 1,45 & 1,22 & 1,04 & 1,02 & 0,88 & 0,76 \\
\hline Valin & 0,82 & 0,70 & 0,62 & 0,66 & 0,57 & 0,50 \\
\hline Arginin & 1,15 & 0,83 & 0,82 & 0,62 & 0,49 & 0,51 \\
\hline \multicolumn{7}{|l|}{ Geschätzte ME } \\
\hline $\mathrm{MJ} / \mathrm{kg}$ & 13,6 & 13,8 & 12,9 & 13,1 & 13,4 & 13,2 \\
\hline $\mathrm{CP} / \mathrm{ME} \quad \mathrm{g} / \mathrm{MJ}$ & 13,4 & 11,3 & 10,6 & 10,7 & 8,8 & 8,1 \\
\hline Lys/ME $\mathrm{g} / \mathrm{MJ}$ & 0,72 & 0,62 & 0,57 & 0,74 & 0,61 & 0,55 \\
\hline
\end{tabular}

Alle Versuchstiere sind am 20. Dezember 1998 im Schlachthof Weißenfels (B.+C. Tönnies Fleischwerk GmbH \& Co. KG) geschlachtet worden. Folgende Merkmale zur Schlachtkörperqualität wurden mit derzeit üblichen Methoden und mit Unterstützung durch das Institut für Tierzucht und Tierhaltung mit Tierklinik der Martin-Luther-Universität Halle-Wittenberg ermittelt: 2-Hälftengewicht, Schlachtausbeute, Magerfleischanteil (mittels FAT-O-MEATer), innere Länge, Rückenspeckdicke (Ø Wider- 
rist, 13./14. Rippe, Ende 3. Kreuzwirbel), Fleischmaß (FMO).

Mit Hilfe des Programms ANOVA/MANOVA im Statistikprogrammpaket STATISTICA (MICROSOFT, 1994) erfolgte die statistische Auswertung der Untersuchungsbefunde. In den Ergebnistabellen sind neben den Mittelwerten die Standardabweichungen $( \pm)$ der Einzelwerte dargestellt. Wesentliche Befunde wurden mittels dreifaktorieller Varianzanalyse ausgewertet. Die Mittelwertvergleiche erfolgten bei statistisch gesicherten F-Werten $(\mathrm{p} \leq 0,05)$ mit dem Newman-Keuls-Testverfahren.

3. Ergebnisse

3.1. Mastdaten

Der Versuch verlief störungsfrei. Die Futterverzehrsdaten zeigen (Tabelle 4), daß alle Futtermischungen gut aufgenommen wurden. Der Futterverzehr der weiblichen Tiere lag im erwarteten Bereich. Der geringere Verzehr der weiblichen Tiere der Versuchsgruppe im 3. Mastabschnitt resultiert aus einer stark verringerten Futteraufnahme eines Tieres im Zeitraum von 5 Tagen. Er war deshalb nicht grupppentypisch und nicht signifikant. Die Börge schöpften ihr Verzehrslimit im zweiten und dritten Mastabschnitt weitgehend aus. Die Varianzanalyse, die wegen der restriktiven Fütterung der Börge ab $60 \mathrm{~kg}$ Lebendmasse eine spezifische Bewertung erfahren muß, ergab, daß der Rohproteingehalt der Futtermischungen keinen signifikanten Einfluß auf den Verzehr besaß. Der Futterverzehr war vor allem vom Mastabschnitt $\left(\mathrm{F}_{\text {exp. }}=\right.$ $564,2)$ und vom Geschlecht $\left(\mathrm{F}_{\text {exp. }}=34,9\right)$ abhängig. Die Differenzen zwischen dem ersten und dem zweiten Mastabschnitt waren bei beiden Fütterungsgruppen und Geschlechtern signifikant, während zwischen dem zweiten und dritten Abschnitt keine signifikanten Unterschiede bestanden.

Tabelle 4

Futterverzehr durch Börge (B) und Sauen (S) in drei Mastabschnitten ( $\mathrm{g} /$ Tier und Tag) (Daily feed intake of barrows (B) and gilts (S) during three fattening periods; $g$ per animal and day)

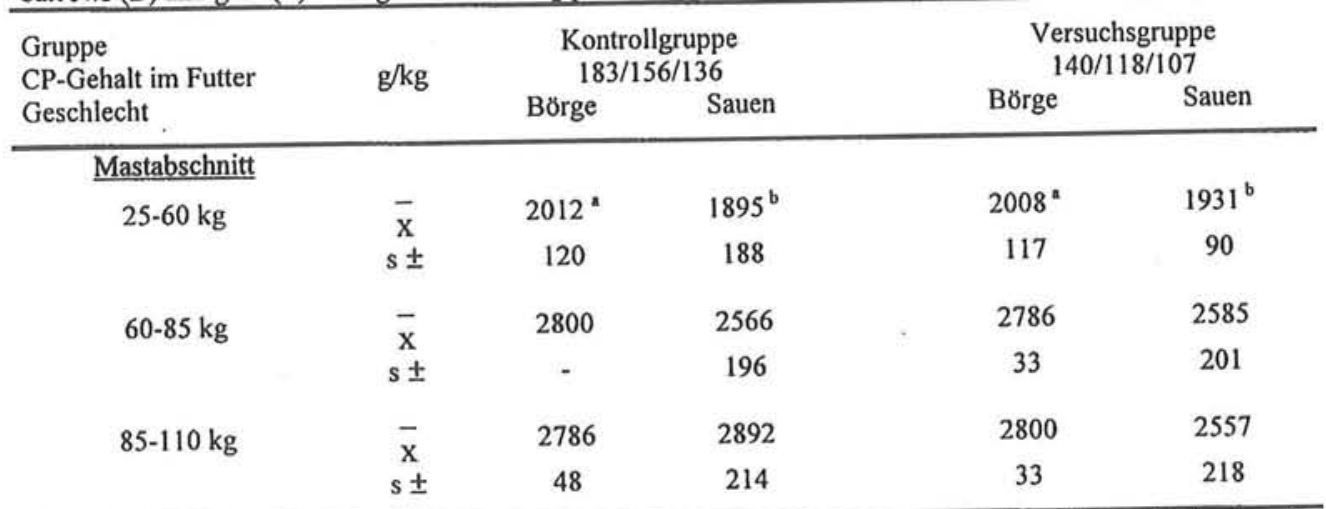

Unterschiedliche Kleinbuchstaben kennzeichnen signifikante Differenzen der Mittelwerte ( $p \leq 0,05$ ), hier nur for den jeweiligen Mastabschnitt zutreffend

Die erreichten täglichen Zunahmen sind in Tabelle 5 ausgewiesen und dokumentieren bei einem Gesamtmittel von $866 \mathrm{~g} /$ Tier ein hohes Niveau bei beiden Fütterungsvari- 
anten. Es bestanden keine signifikanten Unterschiede zwischen den beiden Fütterungsvarianten und zwischen den Geschlechtern. Biostatistisch begründete Zusammenhänge konnten mittels Varianzanalyse nur zwischen den Mastabschnitten ( $\mathrm{F}_{\text {exp. }}=$ 5,9 ; signifikante Differenzen zwischen dem ersten und dritten Mastabschnitt) und hinsichtlich der Wechselwirkung zwischen Mastabschnitten und Geschlechtern ( $F_{\text {exp. }}=$ 27,3) ermittelt werden. Die Börge nahmen durchschnittlich täglich im ersten Mastabschnitt $149 \mathrm{~g}$ mehr und in den beiden folgenden Mastabschnitten 75 bzw. $60 \mathrm{~g}$ weniger zu als die Sauen. Diese Differenzen waren aber nicht signifikant.

Tabelle 5

Lebendmassezunahme von Börgen (B) und Sauen (S) in den drei Mastabschnitten (g/Tier und Tag) (Daily weight gain of barrows (B) and gilts (S) during three fattening periods; $g$ per animal and day)

\begin{tabular}{|c|c|c|c|c|c|}
\hline \multirow{2}{*}{$\begin{array}{l}\text { Gruppe } \\
\text { CP-Gehalt im Futter } \\
\text { Geschlecht }\end{array}$} & \multirow[t]{2}{*}{$\mathrm{g} / \mathrm{kg}$} & \multicolumn{2}{|c|}{$\begin{array}{c}\text { Kontrollgruppe } \\
183 / 156 / 136\end{array}$} & \multicolumn{2}{|c|}{$\begin{array}{c}\text { Versuchsgruppe } \\
140 / 118 / 107\end{array}$} \\
\hline & & Börge & Sauen & Börge & Sauen \\
\hline \multicolumn{6}{|l|}{ Mastabschnitt } \\
\hline \multirow[t]{2}{*}{$25-60 \mathrm{~kg}$} & $\bar{x}$ & 966 & 821 & 970 & 816 \\
\hline & $s \pm$ & 49 & 90 & 61 & 63 \\
\hline \multirow[t]{2}{*}{$60-85 \mathrm{~kg}$} & $\bar{x}$ & 813 & 916 & 825 & 872 \\
\hline & $s \pm$ & 47 & 127 & 52 & 89 \\
\hline \multirow[t]{2}{*}{$85-110 \mathrm{~kg}$} & $\bar{x}$ & 796 & 879 & 816 & 853 \\
\hline & $s \pm$ & 62 & 135 & 36 & 111 \\
\hline
\end{tabular}

Tabelle 6

Futteraufwand je kg Lebendmassezunahme bei Borgen (B) und Sauen (S) in den drei Mastabschnitten (kg) (Feed Consumption per $\mathrm{kg}$ weight gain of barrows (B) and gilts (S) during three fattening periods; $\mathrm{kg}$ )

\begin{tabular}{|c|c|c|c|c|c|}
\hline \multirow{2}{*}{$\begin{array}{l}\text { Gruppe } \\
\text { CP-Gehalt im Futter } \\
\text { Geschlecht }\end{array}$} & \multirow[t]{2}{*}{$\mathrm{g} / \mathrm{kg}$} & \multicolumn{2}{|c|}{$\begin{array}{c}\text { Kontrollgruppe } \\
183 / 156 / 136\end{array}$} & \multicolumn{2}{|c|}{$\begin{array}{c}\text { Versuchsgruppe } \\
140 / 118 / 107\end{array}$} \\
\hline & & Börge & Sauen & Borge & Sauen \\
\hline \multicolumn{6}{|l|}{ Mastabschnitt } \\
\hline \multirow[t]{2}{*}{$25-60 \mathrm{~kg}$} & $\bar{x}$ & 2,09 & 2,33 & 2,08 & 2,38 \\
\hline & $s \pm$ & 0,10 & 0,25 & 0,14 & 0,15 \\
\hline \multirow[t]{2}{*}{$60-85 \mathrm{~kg}$} & $\bar{x}$ & 3,45 & 2,84 & 3,39 & 2,98 \\
\hline & $s \pm$ & 0,20 & 0,37 & 0,23 & 0,26 \\
\hline \multirow[t]{2}{*}{$85-110 \mathrm{~kg}$} & $\bar{x}$ & 3,52 & 3,33 & 3,44 & 3,03 \\
\hline & $s \pm$ & 0,31 & 0,29 & 16 & 0,38 \\
\hline
\end{tabular}

Tabelle 6 informiert über den Futteraufwand je kg Lebendmassezunahme. Er liegt im Gesamtmittel bei $2,78 \mathrm{~kg}$ und ist in Anbetracht des erreichten Niveaus der täglichen Lebendmassezunahmen im zu erwartenden Bereich. Zwischen den Fütterungsvarianten bestehen keine signifikanten Differenzen. Neben den lebendmassebedingten Unterschieden $\left(\mathrm{F}_{\text {exp. }}=274,1\right)$ zwischen den Mastabschnitten traten trotz der restriktiven 
Fütterung der Börge ab $60 \mathrm{~kg}$ Lebendmasse signifikante Differenzen zwischen den Geschlechtern $\left(\mathrm{F}_{\text {exp. }}=18,4\right)$ auf. Im ersten Mastabschnitt benötigten die Börge und in den folgenden Mastabschnitten die weiblichen Tiere weniger Futter je kg Lebendmassezunahme. Diese Differenzen waren aber für die einzelnen Mastabschnitte nicht signifikant.

\section{N-Ausscheidungen}

Die kalkulierten Befunde über die N-Ausscheidungen sind in Tabelle 7 ausgewiesen. Daraus geht hervor, daß durch Reduzierung der Rohproteingehalte in den Futtermischungen von 183/156/136 g/kg (Kontrollgruppe) auf 140/118/107 g/kg (Versuchsgruppe) die N-Ausscheidungen je kg Lebendmassezunahme um 37,5\% verringert werden konnten. Die Abnahmen der N-Ausscheidungen waren von der Fütterungsvariante $\left(\left(F_{\text {exp. }}=399,9\right)\right.$, dem Lebendmassebereich $\left(F_{\text {exp. }}=92,6\right)$ und dem Geschlecht $\left(\mathrm{F}_{\text {exp. }}=27,6\right)$ abhängig. Außerdem bestand zwischen den Mastabschnitten und dem Geschlecht eine Wechselwirkung $\left(F_{\text {exp. }}=37,6\right)$. Bei Einzelbetrachtung der Mastabschnitte waren nur die Differenzen zwischen den Fütterungsgruppen signifikant.

Tabelle 7

$\mathrm{N}$-Ausscheidungen von Börgen und Sauen $(\mathrm{g} / \mathrm{kg}$ Lebendmassezunahme) ( $\mathrm{N}$-excretion of barrows (B) and gilts (S); $\mathrm{g} / \mathrm{kg}$ weight gain)

\begin{tabular}{|c|c|c|c|c|c|}
\hline \multirow{2}{*}{$\begin{array}{l}\text { Gruppe } \\
\text { CP-Gehalt im Futter } \\
\text { Geschlecht }\end{array}$} & \multirow[t]{2}{*}{$\mathrm{g} / \mathrm{kg}$} & \multicolumn{2}{|c|}{$\begin{array}{c}\text { Kontrollgruppe } \\
183 / 156 / 136\end{array}$} & \multicolumn{2}{|c|}{$\begin{array}{c}\text { Versuchsgruppe } \\
140 / 118 / 107\end{array}$} \\
\hline & & Borge & Sauen & Börge & Sauen \\
\hline \multicolumn{6}{|l|}{ Mastabschnitt } \\
\hline \multirow[t]{2}{*}{$25-60 \mathrm{~kg}$} & $\bar{x}$ & $33,7^{b}$ & $40,1^{b}$ & $19,9^{*}$ & $25,0^{\mathrm{A}}$ \\
\hline & $s \pm$ & 2,4 & 6,4 & 2,6 & 2,7 \\
\hline \multirow[t]{2}{*}{$60-85 \mathrm{~kg}$} & $\bar{x}$ & $59,2^{b}$ & $44,5^{b}$ & $37,3^{*}$ & $29,5^{*}$ \\
\hline & $s \pm$ & 4,5 & 8,1 & 3,8 & 4,4 \\
\hline \multirow[t]{2}{*}{$85-110 \mathrm{~kg}$} & $\bar{x}$ & $43,9^{b}$ & $36,2^{b}$ & $28,2^{\prime \prime}$ & $22,6^{\text {A }}$ \\
\hline & $s \pm$ & 5,3 & 5,8 & 1,9 & 5,5 \\
\hline \multirow[t]{2}{*}{$25-110 \mathrm{~kg}$} & $\bar{x}$ & $43,4^{c}$ & $39,8^{b}$ & $26,6^{a}$ & $25,4^{\mathrm{a}}$ \\
\hline & $s \pm$ & 1,8 & 3,1 & 2,1 & 2,8 \\
\hline
\end{tabular}

Unterschiedliche Kleinbuchstaben kennzeichnen signifikante Differenzen der Mittelwerte ( $\leq \leq 0,05$ ), hier nur fur den jeweiligen Mastabschnitt zutreffend

\subsection{Schlachtleistung und Schlachtkörperqualität}

Die an allen in den Versuch einbezogenen Tieren bestimmten Daten über die Schlachtleistung und Schlachtkörperqualität sind für beide Geschlechter getrennt in Tabelle 8 aufgeführt. Bei keinem der erfaßten Parameter bestehen signifikante Unterschiede zwischen den Fütterungsgruppen, auch nicht bei gemeinsamer Auswertung beider Geschlechter. Alle Tiere hatten eine gute Schlachtleistung und Schlachtkörperqualität. Auf Grund des Magerfleischanteils wurde die Mehrzahl der Tiere in die Handelsklasse 
E (beste Klasse) eingestuft. Dabei übertrafen die weiblichen Tiere die Börge, die trotz restriktiver Fütterung ab $60 \mathrm{~kg}$ Lebendmasse mehr Fett ansetzten. Die weiblichen Tiere besaßen signifikant größere Magerfleischanteile $\left(F_{\text {exp. }}=60,8\right)$ und Fleischmaße $\left(F_{\text {exp. }}=6,6\right)$, während ihre Rückenspeckdicke signifikant niedriger $\left(F_{\text {exp. }}=57,1\right)$ lag. Obwohl mittels statistischer Auswertung keine Unterschiede bei der Schlachtkörperqualität zwischen den Fütterungsgruppen ermittelt werden konnten, war bei den Börgen der Versuchsgruppe vergleichsweise zu denen der Kontrollgruppe tendenziell eine geringfügige Abnahme des Magerfleischanteils (um absolut 0,9\%) und Zunahme der Rückenspeckdicke $(0,5 \mathrm{~mm})$ zu beobachten.

Tabelle 8

Schlachtleistung und Schlachtkörperqualität (Slaugther performance and carcass quality

\begin{tabular}{|c|c|c|c|c|c|}
\hline \multirow{2}{*}{$\begin{array}{l}\text { Gruppe } \\
\text { CP-Gehalt im Futter } \\
\text { Geschlecht }\end{array}$} & \multirow[t]{2}{*}{$\mathrm{g} / \mathrm{kg}$} & \multicolumn{2}{|c|}{$\begin{array}{c}\text { Kontrollgruppe } \\
183 / 156 / 136\end{array}$} & \multicolumn{2}{|c|}{$\begin{array}{c}\text { Versuchsgruppe } \\
140 / 118 / 107\end{array}$} \\
\hline & & Börge & Sauen & Børge & Sauen \\
\hline \multirow[t]{2}{*}{ 2-Hălftengewicht } & $\operatorname{kg} \bar{x}$ & 88,0 & 87,5 & 88,7 & 85,1 \\
\hline & $s \pm$ & 1,7 & 5,4 & 3,1 & 4,1 \\
\hline \multirow[t]{2}{*}{ Schlachtausbeute } & $\% \bar{x}$ & 79,8 & 79,6 & 79,7 & 79,6 \\
\hline & $s \pm$ & 0,9 & 0,6 & 0,5 & 0,7 \\
\hline \multirow[t]{2}{*}{ Magerfleischanteil } & $\% \bar{x}$ & $53,5^{*}$ & $57,0^{b}$ & $52,6^{\circ}$ & $57,3^{b}$ \\
\hline & $s \pm$ & 2,2 & 1,3 & 1,8 & 1,7 \\
\hline Handelsklasse ${ }^{*}$ & $\operatorname{kg} \bar{x}$ & 1,8 & 1,1 & 2,2 & 1,2 \\
\hline (EUROP) & $s \pm$ & 0,6 & 0,3 & 0,4 & 0,4 \\
\hline \multirow[t]{2}{*}{ Speckdicke } & $\mathrm{mm} \bar{x}$ & $19,5^{*}$ & $15,5^{b}$ & $20,0^{a}$ & $15,2^{b}$ \\
\hline & $s \pm$ & 25 & 1,6 & 2,1 & 1,5 \\
\hline Fleischmaß & $\mathrm{mm} \overline{\mathrm{x}}$ & $58,9^{\star}$ & $60,6^{b}$ & $58,3^{\star}$ & $63,2^{b}$ \\
\hline (FMO) & $s \pm$ & 5,6 & 5,0 & 3,5 & 4,1 \\
\hline
\end{tabular}

" $\mathrm{E}=1 ; \mathrm{U}=2 ; \mathrm{R}=3$ Unterschiedliche Kleinbuchstaben kennzeichnen signifikante Differenzen der Mittelwerte (p $\leq 0,05$ )

\section{Diskussion}

Die Rohproteingehalte konnten in den sechs geprüten Futtermischungen (zwei Fütterungsgruppen und drei Fütterungsabschnitte) vergleichsweise zum Versuchsplan mit akzeptabler Genauigkeit eingehalten werden. Dagegen wurden die angestrebten Energiedichten von einheitlich 13,0 MJ ME/kg nur annähernd (12,9-13,8 MJ ME/kg)) erreicht. Innnerhalb der jeweiligen Mastabschnitte bestanden Differenzen von 0,5, 0,4 und $0,3 \mathrm{MJ} \mathrm{ME} / \mathrm{kg}$, die aber nicht gleichgerichtet waren. Durch die Unterschiede bei den Energiedichten in den Futtermischungen erfuhren auch die Lysin/EnergieVerhältnisse eine gewisse Beeinflussung, die jedoch zwischen den Fütterungsvarianten in engen Grenzen $(0,72-0,74 ; 0,61-0,62 ; 0,55-57 \mathrm{~g}$ Lysin je MJ ME) blieben. Bei allen 
Varianten wurde somit den diesbezüglich gestellten Anforderungen (0,74, 0,63 und $0,55)$ weitgehend entsprochen. Hervorzuheben ist, daß alle zugrunde gelegten Mindestgehalte an essentiellen Aminosäuren (Tab. 1) in den Futtermischungen (Tab. 3) erreicht bzw. übertroffen worden sind. Auch bei Heranziehung neuerer Empfehlungen (BAKER; 1997, SCHULZ, 1998; NRC 1998) liegen die Lysin/Energie-Verhältnisse sowie die Relationen von Lysin zu den übrigen essentiellen Aminosäuren im geforderten Bereich. Die Versorgung mit nichtessentiellen Aminosäuren bzw. der Gesamtverzehr an Aminosäuren ( $\alpha$-Aminostickstoff) waren, entsprechend den Erkenntnissen von ROTH et al. (1993), wonach Bedarfsrelationen zwischen essentiellen (ohne Arginin) und nichtessentiellen Aminosäuren von 47:53 bestehen, ebenfalls ausreichend. Die vergleichsweise hohen Wachstumsleistungen, ohne Unterschiede zwischen Kontroll- und Versuchsgruppe, und die für die genetische Konstruktion der Versuchstiere gute Schlachtkörperzusammensetzung können gleichfalls als Beleg für eine für das Wachstum ausreichende Aminosäurenversorgung angesehen werden.

Die Empfehlungen der GfE (1987) zur Rohproteinversorgung von Mastschweinen sind bei proteinreduzierter Fütterung kein Maßstab, da sie nur auf einen Lysingehalt von $5 \%$ im Rohprotein orientiert sind. Bei Supplementierung mit freien Aminosäuren sind höhere Konzentrationen an Lysin und analog für andere essentielle Aminosäuren im Protein erreichbar, so daß der Aminosäurenbedarf mit weniger Rohprotein gedeckt werden kann.

Bei Beurteilung der geschlechtsspezifischen Unterschiede der Wachstumsleistungen sowie des Futter- und Energieaufwandes ist die restriktive Fütterung der Börge ab $60 \mathrm{~kg}$ Lebendmasse zu berücksichtigen. Bemerkenswert sind die vergleichsweise geringeren Lebendmassezunahmen der Börge im zweiten und dritten Mastabschnitt, obwohl sie trotz restriktiver Fütterung die weiblichen Tiere im Futterverzehr noch etwas übertrafen. Aus dieser Tatsache resultiert auch der tendenziell höhere Futteraufwand der Börge in diesen Mastabschnitten. Bei Heranziehung aller drei Mastabschnitte bestehen beim Futteraufwand beider Geschlechter (Börge 2,80 kg, Sauen $2,75 \mathrm{~kg} / \mathrm{kg} \mathrm{LMZ)} \mathrm{jedoch} \mathrm{nur} \mathrm{geringfügige} \mathrm{Unterschiede.} \mathrm{Hinsichtlich} \mathrm{des}$ Futteraufwandes konnte auch für die gesamte Mastdauer zwischen Versuchs- und Kontrollgruppe eine weitgehende Übereinstimmung (2,76 kg bzw. $2,79 \mathrm{~kg} / \mathrm{kg} \mathrm{LMZ}$ ) festgestellt werden. Dagegen bestanden beim Energieaufwand zwar relativ kleine, aber signifikante Differenzen (36,51 MJ ME bzw. 37,43 MJ ME/kg LMZ, ca. 2,5\% rel.) zugunsten der Versuchsgruppe. Der niedrigere Energieaufwand der Tiere der Versuchsgruppe resultiert aus einer Abnahme der Stoffwechselbelastung der Tiere infolge geringerer Harnstoffsynthese (BERGNER, 1989; JENTSCH et al., 1991; KIRCHGESSNER et al., 1994; MOEHN und SUSENBETH, 1995; BAKER, 1997). Der vergleichsweise schwache Effekt muß im Zusammenhang mit den bereits relativ niedrigen Proteingehalten der Futtermischungen der Kontrollgruppe gesehen werden.

Die N-Ausscheidungen (Tab. 7) sind zwar Kalkulationswerte, wurden aber für jedes Einzeltier auf der Basis des geschätzten Proteinansatzes sowie der genau erfaßten Proteinaufnahme ermittelt und besitzen, wie frühere Untersuchungen (NONN et al.,1993; KRACHT et al., 1994, NONN und FRANKE, 1998) zeigten, eine akzeptable Übereinstimmung mit Meßwerten aus N-Bilanzbestimmungen. Für praxisrelevante Bedingungen dürfte ihre Aussagekraft ausreichend sein, zumal der ganze Wachstums- 
abschnitt in die Kalkulation einbezogen wird. Nach vorliegender Kalkulation konnten die N-Ausscheidungen für die Gesamtmastdauer bei der Versuchsgruppe vergleichsweise zur Kontrollgruppe um 37,5 \% verringert werden, obwohl die Rohproteingehalte der Futtermischungen der Kontrollgruppe schon vergleichsweise niedrig lagen. Der Produktive Proteinwert (PPW) stieg von 38,5 (KG) auf 50,1 \% (VG). Die für die Versuchsgruppe ermittelten N-Ausscheidungen von $26 \mathrm{~g} / \mathrm{kg}$ Lebendmassezunahme $(2,60 \mathrm{~kg} / 100 \mathrm{~kg} \mathrm{LMZ})$ stellen für den gesamten Mastabschnitt einen extrem niedrigen Wert dar (JONGBLOED et al., 1991; COLE und VAN LUNEN, 1994; KIRCHGESSNER et al., 1994 ; SUSENBETH et al., 1994; KERR et al., 1995; WALZ und PALLAUF, 1995; HAHN und BAKER, 1995; NONN und FRANKE, 1998). Dabei bestanden zwischen den Börgen (KG: 4,34; VG 2,66 kg/100 kg LMZ) und weiblichen Tieren (KG 3,98; VG: $2,54 \mathrm{~kg} / 100 \mathrm{~kg} \mathrm{LMZ}$ ) geringe aber für die gesamte Mastdauer statistisch gesicherte Unterschiede.

Die bestimmten Schlachtkörperqualitätsparameter wurden durch die Reduzierung der Proteinaufnahme bei den weiblichen Tieren nicht beeinträchtigt. Bei den Börgen wurden geringfügige statistisch nicht gesicherte Abnahmen des Magerfleischanteils und Zunahmen der Rückenspeckdicke beobachtet. Sie liegen in einem noch zu tolerierenden Bereich und können ihre Ursache in einer zu knappen Aminosäuren- oder zu reichlichen Energieversorgung haben. Wahrscheinlicher erscheint eine Unterversorgung mit Aminosäuren, die sich stärker auf die Schlachtkörperqualität als auf die Wachstumsleistung auswirkt. Dabei wird die Abnahme des Proteinansatzes teilweise durch einen höheren Fettansatz kompensiert. Solche Beobachtungen machten wir bereits bei unseren früheren Untersuchungen (NONN u. FRANKE, 1998) und sind auch von anderen Versuchsanstellern (RUSSEL et al., 1983; VON ESSEN und GÜNTHER, 1990; ROLING, 1992; SEIWERT 1994; KERR,1995) bekannt. Die Börge reagieren dabei, wie bereits erörtert (NONN und FRANKE, 1998), wegen hormonell bedingter schlechterer intermediärer Proteinverwertung wesentlich deutlicher auf Unter- bzw. suboptimale Versorgung mit bestimmten essentiellen Aminosäuren.

Mit vorliegenden Untersuchungen konnte gezeigt werden, daß bei getreidereichen Futtermischungen bei Supplementierung mit 8 essentiellen Aminosäuren (Lys, Met, Thr, Trp, Leu, Ile, His, Val) die Rohproteingehalte bei dreiphasiger Fütterung in den Mastabschnitten $25-60 \mathrm{~kg}, 60-85 \mathrm{~kg}$ und $85-110 \mathrm{~kg}$ Lebendmasse auf $14 \%, 11,8 \%$ bzw. 10,7 \% gesenkt werden können, ohne Wachstumsleistungen und Schlachtkörperqualität nennenswert zu beeinflussen. Nur bei den Börgen war vergleichsweise zur Kontrollgruppe der Magerfleischanteil um 0,9\% (absolut) geringer und die Rückenspeckdicke um $0,5 \mathrm{~mm}$ höher (beides nicht signifikant). Mit einer solchen Fütterung können die N-Ausscheidungen auf ca. $30 \mathrm{~g} / \mathrm{kg}$ Lebendmassezunahme gesenkt werden. Das bedeutet vergleichsweise zu unserer früheren Versuchsanstellung mit Supplementierung von vier Aminosäuren (Lys, Met, Thr, Trp) und N-Ausscheidungen von etwa $40 \mathrm{~g} / \mathrm{kg}$ Lebendmassezunahme (NONN und FRANKE, 1998) eine Reduzierung um ca. $25 \%$ und vergleichsweise zum gegenwärtigen Praxisstand eine Verringerung um ca. 35 bis $50 \%$.

\section{Danksagung}

Die Autoren bedanken sich bei der Lohmann Animal Health GmbH \& Co. KG, Cuxhaven, 
für die finanzielle Förderung der Untersuchungen und beim Institut für Tierzucht und Tierhaltung mit Tierklinik der Martin-Luther-Universität Halle-Wittenberg für die Unterstützung bei der Erfassung der Schlachtkörperqualitätsdaten.

\section{Literatur}

BAKER, D.H.

Ideal amino acid profiles for swine and poultry and their applications in feed formulation. Biokyowa

Technical Review 9 (1997), Nutri-Querst, Inc., Chesterfield, MO, USA

BASSLER, R.; BUCHHOLZ, H.:

Methodenbuch Band III. Die chemische Untersuchung von Futtermitteln. 3. Ergănzungslieferung, VDLUFA-Verlag Darmstadt (1993)

BERGNER, H.:

N-Stoffwechsel und seine Regelmechanismen. Arch. Anim. Nutr. 39 (1989), 377-392

CHUNG, T.K.; BAKER, D.H.:

Ideal protein for swine and poultry. Biokyowa Technical Review 4 (1992), Nutri-Querst, Inc., Chesterfield, MO, USA

COLE, D..J.A.; VAN LUNEN, T.A.

Ideal amino acid patterns. Pp.99-112 in Amino Acid in Farm Animal Nutrition.J.P.F.D'Mello, ed Wallingford, U. K.: CAB International (1994).

ESSEN, v. B.; GÜNTHER, K.D.:

Erfahrungen mit proteinarmen Getreiderationen in der Schweinemast. Lohmann-Informationen Jan./Febr. 1990, 9-13

GESELLSCHAFT FƯR ERNÄHRUNGSPHYSIOLOGIE (GfE)

(Ausschuß fur Bedarfsnormen) Energie - und Nährstoffbedarf landwirtschaftlicher Nutztiere, Nr. 4

Schweine, DLG-Verlag, Frankfurt/M., 1987, 30-43

JENTSCH, W.; HOFMANN, L.; BEYER, M.:

Untersuchungen zum Energieumsatz wachsender Schweine im Lebendmasseabschnitt von 10-50 kg. 2. Mitt.: Stickstoff- und Energieansatz. Arch. Anim. Nutr. 41 (1991), 623-635

HAHN, J.D.; BAKER, D.H.

Optimum ratio of lysine to threonine, tryptophan, and sulfur amino acids for finishing swine. J. Anim. Sci. 73 (1995), 482-489

JONGBLOED, A.W.; LENIS, N.P.:

Nutrition as a maens to reduce environmental pollution by pigs. 42. Jahrestagung der EVT, 08.-12.

September 1991 in Berlin

KERR, B.J.; Mc KEITH, F.K.; EASTER, R.A.:

Effect on performance and carcass characteristics of nursery to finisher pigs fed reduced crude protein, aminoacidsupplemented diets. J. Anim. Sci. 73 (1995), 433-440

KIRCHGESSNER, M.; MARKERT, W.; ROTH, F.X.:

Einfluß der Lysin- und Energieversorgung auf die N-Bilanz von Mastschweinen. 4. Mitteilung über Bilanzstudien zur Reduzierung der N-Ausscheidungen. J. Anim. Physiol. a Anim. Nutr. 71 (1994), 147 155

KRACHT, W.; MATZKE, W.; JEROCH, H.:

Reduzierung der Stickstoffausscheidung beim wachsenden Schwein zur Minimierung der Umweltbelastung. Mokslines Konferencijos „Gyvulinikysté ir Ekologija“, Kaunas (1994)

MARKERT, W.:

Bilanzstudien beim Schwein zur Reduzierung der Stickstoff-Ausscheidung durch Proteinabsenkung und den Einsatz von Aminosäuren. TU Weihenstephan, Diss., 1992

MARKERT, W.; KIRCHGESSNER, M.; ROTH, F.X.:

Optimale Versorgung mit essentiellen Aminosäuren. 1. Mitteilung uber Bilanzstudien zur Reduzierung der N-Ausscheidungen. J. Anim. Physiol. a Anim. Nutr. 70 (1993), 159-171

MICROSOFT, STATISTICA FOR WINDOWS:

General Conventions and Statistics I. General Anova/Manova 1533-1637, Copryright 1994 by statSoft. Inc. 2325 Est 13 th Street, Tulsa OK 74104

MOEHN, S.; SUSENBETH, A.: Influence of dietary protein content on efficienca of energy utilization in growing pigs. Arch. Anim. Nutr. 47 (1995), 361-372 
NAUMANN, C.; BASSLER, R.:

Methodenbuch Band III: Die chemische Untersuchung von Futtermitteln. Mit 2. Ergänzungslieferung 1982 und 3. Ergănzungslieferung 1993. VDLUFA-Verlag, Darmstadt

NONN, H.; BRETTSCHNEIDER, J.; JEROCH, H.; KOCH, F.:

Reduzierung der N-Ausscheidungen bei Mastschweinen. Kuhn-Archiv 87 (1993), 212-221

NONN, H.; FRANKE, C.:

Zur N-reduzierten Futterung bei Einsatz freier Aminosäuren. Arch. Tierz., Dummerstorf 41 (1998) 5 , 473-488

NATIONAL RESEARCH COUNCIL (NRC)

ROLING, $\mathrm{H}$ :

Nutrient requirements of swine, National Academy Press, Washington, D.C.; 1998, 16-46

Erfolgreich und umweltentlastend futtern. Deutsche Geflugelwirtschaft und Schweineproduktion 21 (1992), 615-618

ROTH, F. X.; MARKERT, W.; KIRCHGESSNER, M.:

Zur optimalen Versorgung mit $\alpha$-Aminostickstoff von Mastschweinen. 2. Mitteilung uber Bilanzstudien zur Reduzierung der N-Ausscheidungen. J. Anim. Physiol. a. Anim. Nutr. 70 (1993), 196-206

ROTH, F. X.; MARKERT, W.; KIRCHGESSNER, M.:

Zum Einfluß der Supplementierung von Niedrig-Protein-Rationen mit nicht-essentiellen Aminosăuren auf die N-Bilanz von Mastschweinen. 3. Mitteilung uber Bilanzstudien zur Reduzierung der NAusscheidungen. J. Anim. Physiol. a. Anim. Nutr. 71 (1994), 76-86

RUSSEL, L. E.; CROMWELL, G.L.; STAHLY, T.S.:

Tryptophan, threonine, isoleucine and methionine supplementation of a $12 \%$ protein, lysineSCHULZ, E: supplemented, corn-soybean meal diet for growing pigs. Anim. Sci. 56 (1983), 1115-1123

Ernährung von Mastschweinen - neue Erkenntnisse und Entwicklungstrends. Proc. 5. Tagung Schweine- und Geflugelernăhrung der Martin-Luther-Universităt Halle-Wittenberg 01.-03.Dez. 1998, 1-15

SEIWERT, C.:

Untersuchungen zum notwendigen Einsatz von kristallinem Tryptophan und Isoleucin in proteinreduzierten Futtermischungen für verschiedene Phasen der Schweinemast. Univ. Bonn, Diss., 1994

SUSENBETH, A.; SCHNEIDER, R.; MENKE, K.H.

The effect of protein and lysine intake on growth and protein retention in pigs. J. Anim. Physiol. a. Anim. Nutr. 71 (1994), 200-207

WALZ, O.P.; PALLAUF, J.:

Protein and phosphorus utilization and carcass quality of pigs kept in metabolic cages from $25-100 \mathrm{~kg}$ weight and fed diets low in protein and phosphorus. In: - VIIth Symposium on Protein Metabolism and Nutrition, Vale de Santarém, Estacâo Zootécnica Nacional. (1995), p. 160

WANG, T.C.; FULLER, M.F.

The optimum dietary amino acid pattern for growing pigs. Anim. Prod. 43 (1990), 155-164

Eingegangen: 21.07 .1999

Akzepticrt: 25.02 .2000

Anschrif der Verfasser

Doz. Dr. habil. HULDREICH NONN, Prof. Dr. habil. Dr. h.c. HEINZ JEROCH

Institut für Ernăhrungswissenschaften der

Martin-Luther-Universităt Halle-Wittenberg

Emil-Abderhalden-Straße 26

D-06108 Halle (Saale) 


\section{TAGUNGSANKÜNDIGUNG}

\section{"Tiergesundheit und Qualitätssicherung in der Schweineproduktion"}

Das diesjährige Frühjahrssymposium der Akademie für Tiergesundheit (AfT) findet am 6. Juni 2000 an der Universität Vechta statt. Namhafte Vertreter aus Wissenschaft, Verwaltung und der Ernährungswirtschaft referieren zu aktuellen Fragen des Tiergesundheitsmanagements unter dem Aspekt der Tiergesundheit und der Qualitätssicherung in der Schweineproduktion.

Anmeldung und weitere Informationen bei der Akademie für Tiergesundheit, Postfach 260164, 53153 Bonn, Tel. $0228 / 3182$ 93, Fax. $0228 / 318298$

KOMMENTAR

\section{TIERARZNEIMITTEL NACH 2000}

Aktuelle Informationen aus der Veterinärmedizin, BfT, Bonn 2000

Die Europäische Union hat eine Mammutaufgabe gelöst: Fristgerecht zum Jahresende 1999 wurden rund 600 Arzneistoffe, die als Tierarzneimittel bei Lebensmitteltieren angewendet werden, auf ihre Sicherheit bewertet und mit Rückstandshöchstwerten (MRLs) versehen. Was im Pflanzenschutz bisher nicht gelungen ist und wozu die USA mehrere Jahrzehnte brauchten, hat die Tierarzneimittelagentur in London in einem gewaltigen Kraftakt geschafft. Unterstützt wurde sie dabei von den Rückstandsfachleuten der Mitgliedstaaten und den Unternehmen, aus denen die notwendigen Daten vorgelegt wurden. Dieser wirkliche Zugewinn für die Verbrauchersicherheit in der EU verdient uneingeschränkte Anerkennung.

Natürlich war diese Leistung nicht ohne Einschnitte möglich. Das Tierarzneimittelspektrum wurde eingeengt. In Spezialindikationen werden die Alternativen geringer, aber insgesamt droht der Gesundheit der Tierbestände in Europa noch keine Gefahr. Gefahren drohen bei den Nischensegmenten. Die hohen Sicherheitsanforderungen haben dazu geführt, dass Tierarten wie Fische, Kaninchen, Ziegen oder Pferde von der Industrie aus ökonomischen Gründen nicht mehr bedient werden können. Hier ist die Innovationskraft der Hersteller bereits überfordert. Deshalb müssen die Sicherheitskonzepte weiter entwickelt werden. Eine Überprüfung des Lebensmittel-Status des Pferdes und die vermehrte Festlegung gewebespezifischer MRLs, die für mehrere Tierarten in gleicher Weise gelten, ist notwendig. Die Erfahrungen belegen, dass zwischen Fleisch von Rind, Schaf, Ziege, Schwein und Kalb rückstandshygienisch keine Unterschiede bestehen. Wir brauchen Regulierung, aber mit Augenmaß.

Aus: BfT Spezial, Nr. 19, S. 4, Februar 2000 